\title{
CENTRAL LIMIT THEOREM FOR GENERALIZED WEIERSTRASS FUNCTIONS
}

\author{
AMANDA DE LIMA AND DANIEL SMANIA
}

\begin{abstract}
Let $f: \mathbb{S}^{1} \rightarrow \mathbb{S}^{1}$ be a $C^{2+\epsilon}$ expanding map of the circle and let $v: \mathbb{S}^{1} \rightarrow \mathbb{R}$ be a $C^{1+\epsilon}$ function. Consider the twisted cohomological equation

$$
v(x)=\alpha(f(x))-D f(x) \alpha(x),
$$

which has a unique bounded solution $\alpha$. We show that $\alpha$ is either $C^{1+\epsilon}$ or nowhere differentiable. Moreover $\alpha$ is nowhere differentiable if and only if $\sigma=\sigma(\phi) \neq 0$, where

$$
\phi(x)=-\left(\frac{v^{\prime}(x)+\alpha(x) D^{2} f(x)}{D f(x)}\right)
$$
\end{abstract}

and

$$
\sigma^{2}(\phi)=\lim _{n \rightarrow \infty} \int\left(\frac{\sum_{j=0}^{n} \phi \circ f^{j}}{\sqrt{n}}\right)^{2} d \mu .
$$

Here $\mu$ is the unique absolutely continuous invariant probability of $f$. We show that if $\alpha$ is nowhere differentiable then

$$
\lim _{h \rightarrow 0} \mu\left\{x: \frac{\alpha(x+h)-\alpha(x)}{\sigma \ell h \sqrt{-\log |h|}} \leq y\right\}=\frac{1}{\sqrt{2 \pi}} \int_{-\infty}^{y} e^{-\frac{t^{2}}{2}} d t .
$$

for some constant $\ell>0$. In particular $\alpha$ is not a Lipschitz continuous function on any subset with positive Lebesgue measure.

\section{INTRODUCTION AND STATEMENT OF THE RESULTS}

In the beginning of the nineteenth century, it was a common belief that a continuous function is differentiable at most of its domain. However, in 1872, Karl Weierstrass gave a stunning example of a function which is everywhere continuous but nowhere differentiable

$$
W(x)=\sum_{k=0}^{\infty} a^{k} \cos \left(b^{k} \pi x\right) .
$$

Here $a$ is a real number with $0<a<1, b$ is an odd integer and $a b>1+3 \pi / 2$. In 1916, G. H. Hardy [17] proved that the function $W$ defined above is continuous and nowhere differentiable if $0<a<1, a b \geq 1$. The constant $b$ does not need to be an integer.

Date: January 8, 2018.

2010 Mathematics Subject Classification. (primary) 37C30, 37C40, 26A15, 26A27, 60F05 (secondary) 37D20, 37A05, 37E05, 26A16, 30H30 .

Key words and phrases. dynamical systems, circle maps, expanding maps, ergodic theory, central limit theorem, Law of iterated logarithm, variance, Livsic, weierstrass function, nowhere differentiable, cohomological equation, Takagi function.

We thank the referees for the very helpful comments. A.L. was partially supported by FAPESP 2010/17419-6 and D.S. was partially supported by CNPq 305537/2012-1, 307617/2016-5 and FAPESP 2017/06463-3. 
There are many contributions on this subject after the introduction of the Weierstrass function. In 1903, T. Takagi [39] presented another example of a nowhere differentiable continuous function, now called Takagi function, given by

$$
T(x)=\sum_{k=0}^{\infty} \frac{1}{2^{k}} \inf _{m \in \mathbb{Z}}\left|2^{k} x-m\right| .
$$

Observe that Weierstrass (in the case $a=b \in \mathbb{N}$ ) and Takagi functions satisfy the cohomological equations

$$
v(x)=W(a x)-\frac{1}{a} W(x), \quad \text { where } \quad v(x)=-\frac{\cos (\pi x)}{a}
$$

and

$$
v(x)=T(2 x)-2 T(x), \quad \text { where } \quad v(x)=-2 \inf _{m \in \mathbb{Z}}|x-m|,
$$

respectively. It turns out these cohomological equations are particular cases of the twisted cohomological equation, that is an essential tool in the study of the smooth perturbations of one-dimensional dynamical systems and the linear response problem (see Baladi and Smania [4] [5] and Lyubich [31], for instance). We can ask if similar results to those by Weiertrass and Takagi holds for more general dynamical systems $f$ and observables $v$. There are some results in this direction by Heurteaux [20], but only when $f$ is a linear dynamical system.

Let us denote by $\mathbb{S}^{1}$ the unit circle, $\mathbb{S}^{1}=\left\{(x, y) \in \mathbb{R}^{2}: x^{2}+y^{2}=1\right\}$. Let $f: \mathbb{S}^{1} \rightarrow \mathbb{S}^{1}$ be an expanding map, that is, there is $\lambda>1$ such that

$$
|D f(x)|>\lambda
$$

for every $x \in \mathbb{S}^{1}$ and let us consider the twisted cohomological equation

$$
v(x)=\alpha(f(x))-D f(x) \alpha(x),
$$

where $v: \mathbb{S}^{1} \rightarrow \mathbb{R}$ and $\alpha: \mathbb{S}^{1} \rightarrow \mathbb{R}$ are bounded functions.

In Baladi and Smania [4] it was proved that the unique bounded function $\alpha$ satisfying (3) is given by the formula

$$
\alpha(y)=-\sum_{n=1}^{\infty} \frac{v\left(f^{n-1}(y)\right)}{D f^{n}(y)} .
$$

Due to the similarity of this expression with the Weierstrass function we will call $\alpha$ a generalized Weierstrass function. Heurteaux [20] considered the case when $f$ is a linear function and $v$ is an almost periodic function defining

$$
F(x)=\sum_{n=0}^{\infty} b^{-n} v\left(b^{n} x\right),
$$

where $1<b<\infty$. This function was called a Weierstrass-type function.

Along this work we will always assume

(A) $f: \mathbb{S}^{1} \rightarrow \mathbb{S}^{1}$ is a $C^{2+\epsilon}$ expanding map, $v: \mathbb{S}^{1} \rightarrow \mathbb{R}$ is a $C^{1+\epsilon}$ function, and $\alpha: \mathbb{S}^{1} \rightarrow \mathbb{R}$ is the generalized Weierstrass function as defined in (4).

Our first results about generalized Weierstrass functions are 
Proposition 1.1 (Zygmund regularity). The function $\alpha$ is in Zygmund class $\Lambda_{1}$, that is, there is $C>0$ such that

$$
|\alpha(x+h)+\alpha(x-h)-2 \alpha(x)| \leq C|h|,
$$

for all $x \in \mathbb{S}^{1}$ and $|h| \leq 1$.

and

Theorem 1.2 (Regularity Dichotomy). One of the following statements holds:

(i) $\alpha$ is of class $C^{1+\epsilon}$;

(ii) $\alpha$ is nowhere differentiable.

Heurteaux [20] proved a similar result for Weierstrass-type functions, when $f$ is a linear dynamical system. In the last section we give a very short proof of this result for non-linear dynamical systems. The above results are not our main results, however most of this work is dedicated to understand when the cases in Theorem 1.2 occurs, providing an easy way to verify on which case a given $v$ and $\alpha$ fit and also to give a more precise understanding of the regularity of $\alpha$ in the second case. Let

$$
\phi(x)=-\left(\frac{v^{\prime}(x)+\alpha(x) D^{2} f(x)}{D f(x)}\right) .
$$

Note that by Proposition 1.1, the function $\phi$ is $\epsilon$-Hölder. Since $f$ is an $C^{2}$ expanding map of the circle, $f$ admits a unique invariant probability that is absolutely continuous with respect to the Lebesgue measure $\mu=\rho m$ (see for instance [40]), where its density $\rho$ is a Hölder function. We show in Lemma 2.7 that

$$
\int \phi d \mu=0 \text {. }
$$

Let us consider

$$
\sigma^{2}(\phi)=\lim _{n \rightarrow \infty} \int\left(\frac{\sum_{j=0}^{n} \phi \circ f^{j}}{\sqrt{n}}\right)^{2} d \mu .
$$

We have the following

Theorem 1.3. We have $\sigma(\phi)=0$ if and only if $\alpha$ is of class $C^{1+\epsilon}$.

Corollary 1.4. The function $\alpha$ is nowhere differentiable if and only if there is a periodic point $x$ of $f$ such that

$$
\sum_{i=0}^{p-1} \phi \circ f^{i}(x) \neq 0 .
$$

Here $p$ is the period of $x$.

Remark 1.5. If $f$ is the linear map $f(x)=b x$ then $\phi=-v^{\prime} / b$. In this case Heurteaux [20] proved that either

i. $\phi(0) \neq 0$, or

ii. $\phi(0)=0, v$ is not constant and 0 is a global extremum of $v$,

are sufficient conditions for $\alpha$ to be nowhere differentiable. This criterion was generalized to Weierstrass functions in higher dimensions by Donaire, Llorente and Nicolau [12]. The sufficiency of (i) follows from Corollary 1.4 in the setting of 1-periodic $C^{1+\epsilon}$ functions $v$. 
Denote

$$
L=\int \log |D f| d \mu>0, \ell=\frac{1}{\sqrt{L}} .
$$

One can ask about the regularity of $\alpha$ when it is nowhere differentiable. We show a Central Limit Theorem for the modulus of continuity of the function $\alpha$.

Theorem 1.6 (Central Limit Theorem for the modulus of continuity). If $\sigma(\phi) \neq 0$ (that is, $\alpha$ is nowhere differentiable) we have

$$
\lim _{h \rightarrow 0} \mu\left\{x \in \mathbb{S}^{1}: \frac{\alpha(x+h)-\alpha(x)}{\sigma(\phi) \ell h \sqrt{-\log |h|}} \leq y\right\}=\frac{1}{\sqrt{2 \pi}} \int_{-\infty}^{y} e^{-\frac{t^{2}}{2}} d t .
$$

Corollary 1.7. If $\alpha$ is not $C^{1+\epsilon}$ then $\alpha$ is nowhere differentiable and it is not a lipschitzian function on any measurable subset $A \subset \mathbb{S}^{1}$ with $\mu(A)>0$.

We also have

Theorem 1.8 (Law of iterated logarithm for the modulus of continuity). If $\sigma(\phi) \neq$ 0 (that is, $\alpha$ is nowhere differentiable) we have

$$
\limsup _{h \rightarrow 0} \frac{\alpha(x+h)-\alpha(x)}{h \sqrt{-2 \log |h| \log \log (-\log |h|)}}=\sigma(\phi) \ell .
$$

and

$$
\liminf _{h \rightarrow 0} \frac{\alpha(x+h)-\alpha(x)}{h \sqrt{-2 \log |h| \log \log (-\log |h|)}}=-\sigma(\phi) \ell .
$$

for $m$-almost every point $x$.

Gamkrelidze (see [14] and [15]) proved that the Weierstrass and Takagi functions satisfy the Central Limit Theorem and the law of iterated logarithm for the modulus of continuity. Theorems 1.6 and 1.8 are far more general results.

Dynamical systems with a fair amount of hyperbolicity often have remarkable probabilistic properties. Indeed in many cases a sufficiently smooth observable satisfies the Central Limit Theorem (CLT) and the Law of Iterated Logarithm. We also have Poisson Limit Theorems in this setting (see Coelho and Collet [10] and Denker, Gordin, and Sharova [11]). For instance, the CLT holds for $C^{2}$ expanding maps on circle with sufficiently regular observables, and indeed the same holds for piecewise expanding maps on the interval (see Keller [23] and Hofbauer and Keller [21]). Theorems 1.6 and 1.8 fits well with these probabilistic results.

On the other hand note that if it is true that a fairly wide class of dynamical systems have observables that satisfies CLT (see Burton and Denker[9]), if the dynamical system does not have a hyperbolic behaviour (as irrational rotations on the circle) and/or we consider low regularity observables, the Central Limit Theorem often fails in quite a striking way for "typical" continuous observables (see Liardet and Volný [28]).

Indeed for rotations on the circle $f$ with diophantine rotation number and $C^{k}$ functions $v$ with zero average and $k$ large enough, the solution $\alpha$ of the cohomological equation (3) exists and it is always smooth (see for instance Herman [18][19] and the references therein).

Remark 1.9. Corollary 1.4 implies that for a fixed $C^{2+\epsilon}$ expanding map and a generic (in the topological sense) $C^{1+\epsilon}$ function $v$ we have that (7) and (8) hold. This resembles the Lévy's modulus of continuity theorem [27] that gives a CLT 
and law of iterated logarithm for the modulus of the continuity of a typical (in the measure-theoretical sense) path in Wiener process.

Remark 1.10. One should compare Theorem 1.8 with Theorem 1 in Anderson and Pitt [3, Theorem 4.1] that claims that

$$
g(x)=\limsup _{h \rightarrow 0} \frac{|\alpha(x+h)-\alpha(x)|}{|h| \sqrt{-\log |h| \log \log (-\log |h|)}}
$$

is an essentially bounded function for every function $\alpha$ in the Zygmund class.

Remark 1.11. A Bloch function on the unit disk $\mathbb{D}$ is a holomorphic function $\beta: \mathbb{D} \rightarrow \mathbb{C}$ such that

$$
\sup _{z \in \mathbb{D}}\left|\beta^{\prime}(z)\right|\left(1-|z|^{2}\right)<\infty .
$$

Primitives of Bloch functions extend continuously to $\mathbb{S}^{1}$ as functions of Zygmund's class (see Anderson, Clunie and Pommerenke [1] and Anderson and Pitt [2] ). There is a long line of studies of probabilistic-like properties of those functions, and Makarov's law of iterated logarithm [32] is one of the most famous results on this topic. Indeed many authors discovered various quantities associated to a Bloch function that resembles the role of variance in the corresponding probabilistic results. It turns out that for certain "dynamically defined" Bloch functions (see Ivrii [22]) all those variances coincide and the variance has a dynamical interpretation (Przytycki, Urbański and Zdunik [36][37]. See also McMullen [33]and Ivrii [22]). That is the case of Bloch functions with a primitive whose extension to $\mathbb{S}^{1}$ is a function $\alpha$ as in this work, when the variance is $\sigma(\phi) \ell$. So Theorem 1.8 seems to offer yet another way to define variance of a Bloch function on the unit disk, but this time just in terms of its primitive. If $\alpha$ is the primitive of a Bloch function $\beta$ in the unit circle, we define its boundary variance $\sigma_{b}(\beta)$ as

$$
\sigma_{b}(\beta)=\left\|\limsup _{h \rightarrow 0} \frac{|\alpha(x+h)-\alpha(x)|}{|h| \sqrt{-\log |h| \log \log (-\log |h|)}}\right\|_{L^{\infty}\left(\mathbb{S}^{1}\right)} .
$$

\section{Relating the Newton quotient of $\alpha$ with the Birkhoff sum of $\phi$}

In order to prove Theorem 1.6, in this section we will relate the study of the Newton quotients of $\alpha$

$$
\frac{\alpha(x+h)-\alpha(x)}{h}
$$

to the study of the Birkhoff sums

$$
\sum_{i=0}^{N} \phi \circ f^{i}(x) .
$$

Remark 2.1. Suppose that the topological degree of $f$ is $d$. To simplify the notation, we will replace $f, v$ and $\alpha$ by its lifts with respect to the covering $\pi(t)=$ $(\cos (2 \pi t), \sin (2 \pi t))$. That is, we will see $f$ as an expanding function $f: \mathbb{R} \mapsto \mathbb{R}$ satisfying $f(x+1)=f(x)+d$ and $v$ and $\alpha$ as 1-periodic functions $v: \mathbb{R} \rightarrow \mathbb{R}$ and $\alpha: \mathbb{R} \rightarrow \mathbb{R}$.

Definition 2.2. Given $h$ such that $0<|h|<1$ and $x \in \mathbb{S}^{1}$, let $N(x, h)$ be the unique integer such that

$$
\frac{1}{\left|D f^{N(x, h)+1}(x)\right|} \leq|h|<\frac{1}{\left|D f^{N(x, h)}(x)\right|} .
$$


The main result of this section is

Proposition 2.3. Let $N(x, h)$ be as defined in (9). Then

$$
\alpha(x+h)-\alpha(x)=h \sum_{i=0}^{N(x, h)-1} \phi\left(f^{i}(x)\right)+O(h),
$$

where

$$
\phi(x)=-\left(\frac{v^{\prime}(x)}{D f(x)}+\frac{\alpha(x) D^{2} f(x)}{D f(x)}\right) .
$$

Before proving Proposition 2.3 we will need some lemmas. The following is a quite familiar bounded distortion estimate.

Lemma 2.4 (Bounded Distortion). Denote also by $f$ be the lift to $\mathbb{R}$ of the function $f$. Then there is $C>0$ such that for all $n \in \mathbb{N}$

$$
\left|\frac{D f^{n}(x)}{D f^{n}(y)}\right| \leq e^{C\left|f^{n}(x)-f^{n}(y)\right|} .
$$

The following lemma is an easy consequence of Lemma 2.4.

Lemma 2.5. There exists $C>1$ such that for every $x \in \mathbb{R}$ and $h$ satisfying $0<|h|<1$ we have

$$
\frac{1}{C} \leq\left|f^{N(x, h)}[x, x+h]\right| \leq C .
$$

Lemma 2.6. There exists $C>0$ such that for every $h$ satisfying $0<|h|<1$ and for every $j \leq n \leq N(x, h)$

$$
\left|f^{n}(a)-f^{n}(b)\right| \leq \frac{C}{\lambda^{N(x, h)-n}},
$$

and

$$
\left|\frac{D f^{n-j}\left(f^{j} a\right)}{D f^{n-j}\left(f^{j} b\right)}-1\right| \leq \frac{C}{\lambda^{N(x, h)-n}},
$$

for every $a, b \in[x, x+h]$ and $\lambda$ is as in (2).

Proof. It follows from Lemma 2.4 and Lemma 2.5.

Proof of Proposition 2.3. By Lemma 2.6, for every $y \in[x, x+h]$ we have

$$
\begin{aligned}
\alpha(y) & =-\sum_{n=1}^{N(x, h)} \frac{v\left(f^{n-1}(y)\right)}{D f^{n}(y)}-\frac{1}{D f^{N(x, h)}(y)} \sum_{n>N(x, h)}^{\infty} \frac{v\left(f^{n-1}(y)\right)}{D f^{n-N(x, h)}\left(f^{N(x, h)}(y)\right)} \\
& =-\sum_{n=1}^{N(x, h)} \frac{v\left(f^{n-1}(y)\right)}{D f^{n}(y)}+O(h),
\end{aligned}
$$


since by Definition 2.2, Lemmas 2.4 and 2.5

$$
\begin{aligned}
& \left|\frac{1}{D f^{N(x, h)}(y)} \sum_{n>N(x, h)}^{\infty} \frac{v\left(f^{n-1}(y)\right)}{D f^{n-N(x, h)}\left(f^{N(x, h)}(y)\right)}\right| \\
& \leq\left|\frac{1}{D f^{N(x, h)}(x)}\right|\left|\frac{D f^{N(x, h)}(x)}{D f^{N(x, h)}(y)} \sum_{n>N(x, h)}^{\infty} \frac{v\left(f^{n-1}(y)\right)}{D f^{n-N(x, h)}\left(f^{N(x, h)}(y)\right)}\right| \\
& \leq \frac{|h|}{\lambda} e^{C^{2}} \sum_{i=1}^{\infty} \frac{|v|_{\infty}}{\lambda^{i}} .
\end{aligned}
$$

So by the Mean Value Theorem there is $\theta \in[x, x+h]$ such that

$$
\begin{aligned}
& \alpha(x+h)-\alpha(x) \\
& =-h \sum_{n=1}^{N(x, h)} \frac{D v\left(f^{n-1}(\theta)\right) D f^{n-1}(\theta) D f^{n}(\theta)-D^{2} f^{n}(\theta) v\left(f^{n-1}(\theta)\right)}{\left[D f^{n}(\theta)\right]^{2}}+O(h) \\
& =-h \sum_{n=1}^{N(x, h)}\left[\frac{D v\left(f^{n-1}(\theta)\right)}{D f\left(f^{n-1}(\theta)\right)}-\frac{D^{2} f^{n}(\theta) v\left(f^{n-1}(\theta)\right)}{\left[D f^{n}(\theta)\right]^{2}}\right]+O(h)
\end{aligned}
$$

Note that

$$
\begin{aligned}
\sum_{n=1}^{N(x, h)} \frac{D^{2} f^{n}(\theta) v\left(f^{n-1}(\theta)\right)}{\left[D f^{n}(\theta)\right]^{2}} & =\sum_{n=1}^{N(x, h)} \sum_{j=0}^{n-1} \frac{D^{2} f\left(f^{j}(\theta)\right) v\left(f^{n-1}(\theta)\right)}{D f\left(f^{j}(\theta)\right) D f^{n-j}\left(f^{j}(\theta)\right)} \\
& =\sum_{j=0}^{N(x, h)-1} \frac{D^{2} f\left(f^{j}(\theta)\right)}{D f\left(f^{j}(\theta)\right)} \sum_{n=j+1}^{N(x, h)} \frac{v\left(f^{n-j-1}\left(f^{j}(\theta)\right)\right)}{D f^{n-j}\left(f^{j}(\theta)\right)} \\
& =\sum_{j=0}^{N(x, h)-1} \frac{D^{2} f\left(f^{j}(\theta)\right)}{D f\left(f^{j}(\theta)\right)} \sum_{k=1}^{N(x, h)-j} \frac{v\left(f^{k-1}\left(f^{j}(\theta)\right)\right)}{D f^{k}\left(f^{j}(\theta)\right)} \\
& =\sum_{j=0}^{N(x, h)-1} \frac{D^{2} f\left(f^{j}(\theta)\right)}{D f\left(f^{j}(\theta)\right)}\left[-\alpha\left(f^{j}(\theta)\right)+\frac{\alpha\left(f^{N(x, h)}(\theta)\right)}{D f^{N(x, h)-j}\left(f^{j}(\theta)\right)}\right] .
\end{aligned}
$$

and

$$
\sum_{j=0}^{N(x, h)-1}\left|\frac{D^{2} f\left(f^{j}(\theta)\right)}{D f\left(f^{j}(\theta)\right)} \frac{\alpha\left(f^{N(x, h)}(\theta)\right)}{D f^{N(x, h)-j}\left(f^{j}(\theta)\right)}\right| \leq \sum_{j=0}^{N(x, h)-1} \frac{C}{\lambda^{N(x, h)-j}} \leq \frac{C \lambda}{\lambda-1} .
$$

By (11), (12) and (13) we conclude that

$$
\alpha(x+h)-\alpha(x)=h \sum_{j=0}^{N(x, h)-1} \phi\left(f^{j}(\theta)\right)+O(h)
$$

By Baladi and Smania [6] we know that $\alpha$, and consequently $\phi$ is a $\gamma$-Hölder function for every $\gamma \in(0,1)$, so Lemma 2.6 easily implies that

$$
\sum_{j=0}^{N(x, h)-1}\left(\phi\left(f^{j}(\theta)\right)-\phi\left(f^{j}(x)\right)\right)=O(1) .
$$

This concludes the proof. 
Proof of Proposition 1.1. By Proposition 2.3,

$$
\alpha(x+h)-\alpha(x)=-\sum_{n=1}^{N(x, h)} \frac{v^{\prime}\left(f^{n-1}(x)\right)}{D f\left(f^{n-1}(x)\right)}+\frac{\alpha\left(f^{n-1}(x)\right) D^{2} f\left(f^{n-1}(x)\right)}{D f\left(f^{n-1}(x)\right)} h+O(h) .
$$

And

$$
\alpha(x-h)-\alpha(x)=\sum_{n=1}^{N(x, h)} \frac{v^{\prime}\left(f^{n-1}(x)\right)}{D f\left(f^{n-1}(x)\right)}+\frac{\alpha\left(f^{n-1}(x)\right) D^{2} f\left(f^{n-1}(x)\right)}{D f\left(f^{n-1}(x)\right)} h+O(h) .
$$

Therefore,

$$
|\alpha(x+h)+\alpha(x-h)-2 \alpha(x)| \leq K|h|,
$$

which completes the proof.

Lemma 2.7. Let

$$
\phi(x)=-\left(\frac{v^{\prime}(x)+\alpha(x) D^{2} f(x)}{D f(x)}\right) .
$$

Then

$$
\int \phi d \mu=0
$$

where $\mu$ is the unique absolutely continuous invariant probability of $f$.

Proof. Let $\mu_{f}$ be the unique invariant probability of $f$ that is absolutely continuous with respect to the Lebesgue measure. Note that the function

$$
(v, f) \mapsto \int\left(\frac{v^{\prime}(x)+\alpha(x) D^{2} f(x)}{D f(x)}\right) d \mu_{f}
$$

is continuous considering the $C^{1} \times C^{2}$ topology on its domain. This easily follows from the definition of $\alpha$ and the fact that

$$
f \mapsto \mu_{f}
$$

is continuous considering the strong topology on the dual space $\left(C^{0}\right)^{\star}\left(\mathbb{S}^{1}\right)$ (we believe this is a folklore result in the setting of expanding maps on the circle. See Keller and Liverani [24] for references and stronger results). Since $C^{\infty}\left(\mathbb{S}^{1}\right)$ is dense in $C^{k}\left(\mathbb{S}^{1}\right)$, for every finite $k$, it is enough to show (14) when $v \in C^{\infty}$ and $f$ is a $C^{\infty}$ expanding map on the circle. Since $f: \mathbb{R} \rightarrow \mathbb{R}$ is expanding and satisfies $f(x+1)=f(x)+d$ and $v: \mathbb{R} \rightarrow \mathbb{R}$ is 1 -periodic we have $f_{t}=f+t v$ is a family of $C^{\infty}$ expanding maps on $\mathbb{R}$ that induces expanding maps on the circle, provided that $t$ is small enough. We will use the same notation $f_{t}$ for these maps on the circle. Note that $\left.\partial_{t} f_{t}(x)\right|_{t=0}=v(x)$. Since expanding maps of the circle are structurally stable, there is a family of conjugacies $h_{t}$ satisfying $f_{t} \circ h_{t}=h_{t} \circ f$, with $h_{0}(x)=x$. We have $\left.\partial_{t} h_{t}\right|_{t=0}=\alpha(x)$ (see the proof of Theorem 1 in Baladi and Smania [5]). Indeed one can show that

$$
\partial_{t} h_{t}(x)=\alpha_{t}\left(h_{t}(x)\right)
$$

where

$$
\alpha_{t}(x)=-\sum_{n=1}^{\infty} \frac{v\left(f_{t}^{n-1}(x)\right)}{D f_{t}^{n}(x)} .
$$

By Proposition 1.1 there is $C$ such that for every $t$ small enough

$$
\left|\alpha_{t}(x+h)+\alpha_{t}(x-h)-2 \alpha_{t}(x)\right| \leq C|h| .
$$


We can conclude that there exists $C>0$ such that

$$
\left|\alpha_{t}(x)-\alpha_{t}(y)\right| \leq C|x-y|^{\epsilon} .
$$

By (15) and Reimann [38, proof of Proposition 4] for every $\delta>0$ small there exists $C>0$ such that

$$
\left|h_{t}(x)-h_{t}(y)\right| \leq C|x-y|^{1-\delta},
$$

provided that $t$ is small enough. By Baladi and Smania [4, Eqs. (14) and (15)] for each $x$ the map $t \mapsto h_{t}(x)$ is twice differentiable and $\partial_{t}^{2} h_{t}(x)$ satisfies

$$
\partial_{t}^{2} h_{t}(x)=\beta_{t}\left(h_{t}(x)\right),
$$

where

$$
\beta_{t}(x)=-\sum_{n=1}^{\infty} \frac{w_{t}\left(f_{t}^{n-1}(x)\right)}{D f_{t}^{n}(x)}
$$

with

$$
w_{t}(x)=\partial_{t}^{2} f_{t}(x)+2 \partial_{x, t}^{2} f_{t}(x) \alpha_{t}(x)+\partial_{x x}^{2} f_{t}(x) \alpha_{t}^{2}(x) .
$$

Note that $w_{t} \in C^{\epsilon}$ and their Hölder norm is uniformly bounded provided that $t$ is small enough. By Baladi and Smania [6, Proposition 2.3] (indeed here we have a far simpler situation, once $f$ is smooth everywhere) there exists $C>0$ such that

$$
\left|\beta_{t}(x)-\beta_{t}(y)\right| \leq C|x-y|^{\epsilon} .
$$

We claim that the curve $t \mapsto h_{t}$ is a differentiable curve at $t=0$ in the Banach space $C^{\epsilon^{\prime}}$ of $\epsilon^{\prime}$-Hölder functions, for every $\epsilon^{\prime} \in(0, \epsilon)$, and its derivative at $t=0$ is the function $\alpha$. Indeed note that

$$
\begin{aligned}
h_{t}(x)-x-t \alpha(x) & =\left.\int_{0}^{t} \partial_{t} h_{t}(x)\right|_{t=a}-\alpha(x) d a \\
& =\left.\int_{0}^{t} \partial_{t} h_{t}(x)\right|_{t=a}-\left.\partial_{t} h_{t}(x)\right|_{t=0} d a \\
& =\left.\int_{0}^{t} \int_{0}^{a} \partial_{t}^{2} h_{t}(x)\right|_{t=b} d b d a \\
& =\int_{0}^{t} \int_{0}^{a} \beta_{b}\left(h_{b}(x)\right) d b d a .
\end{aligned}
$$

Thus, if $r_{t}(x)=h_{t}(x)-x-t \alpha(x)$ we have by (16), (17) and (18)

$$
\begin{aligned}
\frac{\left|r_{t}(x)-r_{t}(y)\right|}{\left.|x-y|\right|^{\prime}} & \leq \int_{0}^{t} \int_{0}^{a} \frac{\mid \beta_{b}\left(h_{b}(x)\right)-\beta_{b}\left(h_{b}(y) \mid\right.}{\mid x-y \epsilon^{\epsilon^{\prime}}} d b d a \\
& \leq \frac{C^{\epsilon}}{2} t^{2} .
\end{aligned}
$$

This proves the claim. Consider a family of potentials

$$
\psi_{t}(x)=-\log \left|D f_{t}\left(h_{t}(x)\right)\right| .
$$

Using that $t \mapsto h_{t}$ is differentiable at $t=0$ one can prove, with an argument similar to that used to prove that $h_{t}$ is differentiable at $t=0$, that the map

$$
t \mapsto \psi_{t}
$$

is differentiable considering the Banach space of $\epsilon^{\prime}$-Hölder functions on its image, with $\epsilon^{\prime}<\epsilon$. Baladi and Smania [6] did something similar considering the space of 
$p$-bounded variations on the image, with $p$ large. Note that $P\left(f, \psi_{t}\right)=0$ for every $t$, where $P(f, \psi)$ denotes the topological pressure of $\psi$ with respect to $f$. Therefore,

$$
\left.\partial_{t} P\left(f, \psi_{t}\right)\right|_{t=0}=0 \text {. }
$$

The topological pressure with respect to $f$ is a differentiable function on the Banach space of $\epsilon^{\prime}$-Hölder functions and by classical arguments of thermodynamic formalism (see Parry and Pollicott [34]), we have

$$
\left.\partial_{t} P\left(f, \psi_{t}\right)\right|_{t=0}=\left.\int \partial_{t} \psi_{t}\right|_{t=0} d \mu=-\int \frac{v^{\prime}(x)+D^{2} f(x) \alpha(x)}{D f(x)} d \mu=\int \phi d \mu,
$$

where $\mu$ is the equilibrium state of $f$ with respect to $-\log |D f|$, that is, the unique absolutely continuous invariant probability of $f$.

Now we can prove Theorem 1.3.

Proof of Theorem 1.3. If $\sigma^{2}=0$ then by Proposition 4.12 in Parry and Pollicott [34] (see also Proposition 6.1 from Broise [8]), there is a $C^{\epsilon}$-function $u: \mathbb{S}^{1} \rightarrow \mathbb{R}$ such that

$$
\phi=u \circ f-u \text {. }
$$

Then there is $C>0$ such that

$$
\sup _{n, x}\left|\sum_{i=0}^{n} \phi\left(f^{i}(x)\right)\right| \leq C .
$$

And by Proposition 2.3

$$
|\alpha(x+h)-\alpha(x)| \leq\left|\sum_{i=0}^{N(x, h)} \phi\left(f^{i}(x)\right) h\right|+O(h) \leq C|h| .
$$

Therefore $\alpha$ is a lipschitzian function and we can differentiate it at almost every point. Then, differentiating (3), we obtain:

$$
v^{\prime}(x)=\alpha^{\prime}(f(x)) D f(x)-D^{2} f(x) \alpha(x)-D f(x) \alpha^{\prime}(x) .
$$

Therefore,

$$
\alpha^{\prime}(f(x))-\alpha^{\prime}(x)=\frac{v^{\prime}(x)+D^{2} f(x) \alpha(x)}{D f(x)}=\phi(x) .
$$

Since $\phi(x)=u \circ f-u$, it follows that

$$
\left(\alpha^{\prime}-u\right) \circ f(x)=\left(\alpha^{\prime}-u\right)(x) .
$$

Since $f$ is ergodic, we can conclude that there is a constant $k$ such that $\alpha^{\prime}=u+k$. Therefore $\alpha$ is of class $C^{1+\epsilon}$. Reciprocally, if $\alpha$ is of class $C^{1+\epsilon}$, we can differentiate (3). Thus,

$$
v^{\prime}(x)=\alpha^{\prime}(f(x)) D f(x)-D^{2} f(x) \alpha(x)-D f(x) \alpha^{\prime}(x) .
$$

Therefore,

$$
\alpha^{\prime}(f(x))-\alpha^{\prime}(x)=\frac{v^{\prime}(x)+D^{2} f(x) \alpha(x)}{D f(x)} .
$$

Let $p$ be a periodic point of $f$, that is, $f^{n}(p)=p$. Then

$$
\sum_{j=0}^{n-1} \phi\left(f^{j}(p)\right)=\alpha^{\prime}\left(f^{n}(p)\right)-\alpha^{\prime}(p)=\alpha^{\prime}(p)-\alpha^{\prime}(p)=0 .
$$


Therefore, by Livsic Theorem ( Livsic [30]. See also Parry and Pollicott [34]), there is a $\epsilon$-Hölder function $u$ such that

$$
\phi=u \circ f-u .
$$

Hence, as we can see in Proposition 4.12 in Parry and Pollicott [34] (see also Proposition 6.1 from Broise [8]), $\sigma^{2}=0$.

Proof of Corollary 1.4. By Livsic [30], there exists $u \in C^{\epsilon}$ such that $\phi=u \circ f-u$ if and only if for every periodic point $x$ we have

$$
\sum_{i=0}^{p-1} \phi\left(f^{i}(x)\right)=0
$$

where $p$ is the period of $x$. But by Proposition 4.12 in Parry and Pollicott [34] (see also Proposition 6.1 from Broise [8]) such $u$ exists if and only if $\sigma^{2}=0$. Now we can apply Theorem 1.3 to conclude the proof.

\section{Proof of Central Limit Theorem for the modulus of Continuity} OF $\alpha$

We are going to need the following

Lemma 3.1. For $\mu$-a.e. $x \in \mathbb{S}^{1}$,

$$
\lim _{h \rightarrow 0} \frac{N(x, h)}{-\log |h|}=\frac{1}{L}
$$

where $L:=\int \log |D f| d \mu$ is the Lyapunov exponent of $f$.

Proof. By Ergodic Birkhoff's Theorem, for $\mu$-a.e. $x \in \mathbb{S}^{1}$

$$
\begin{aligned}
\lim _{n \rightarrow \infty} \frac{1}{n} \log \left|D f^{n}(x)\right| & =\lim _{n \rightarrow \infty} \frac{1}{n} \log \prod_{j=0}^{n-1}\left|D f\left(f^{j}(x)\right)\right| \\
& =\lim _{n \rightarrow \infty} \frac{1}{n} \sum_{j=0}^{n-1} \log \left|D f\left(f^{j}(x)\right)\right|=\int \log |D f(x)| d \mu=L .
\end{aligned}
$$

By (9) we have

Therefore,

$$
\left|D f^{N(x, h)}(x)\right| \leq \frac{1}{|h|} \leq\left|D f^{N(x, h)+1}(x)\right|
$$

$$
\frac{1}{N(x, h)} \log \left|D f^{N(x, h)}(x)\right| \leq \frac{1}{N(x, h)} \log \frac{1}{|h|} \leq \frac{1}{N(x, h)} \log \left|D f^{N(x, h)+1}(x)\right| .
$$

Hence, for $\mu$-a.e. $x \in \mathbb{S}^{1}$,

$$
\frac{1}{N(x, h)} \log \frac{1}{|h|} \stackrel{N(x, h) \rightarrow \infty}{\longrightarrow} \int \log |D f(x)| d \mu=L
$$

Proof of Theorem 1.6. It is enough to prove that for every sequence $h_{n} \underset{n \rightarrow \infty}{\longrightarrow} 0$, we have

$$
\lim _{n \rightarrow \infty} \mu\left\{x: \frac{\alpha\left(x+h_{n}\right)-\alpha(x)}{\sigma \ell h_{n} \sqrt{-\log \left|h_{n}\right|}} \leq y\right\}=\frac{1}{\sqrt{2 \pi}} \int_{-\infty}^{y} e^{-\frac{t^{2}}{2}} d t .
$$


By Proposition 2.3,

$$
\alpha\left(x+h_{n}\right)-\alpha(x)=h_{n} \sum_{i=1}^{N\left(x, h_{n}\right)} \phi\left(f^{i-1}(x)\right)+O\left(h_{n}\right) .
$$

Since

$$
\frac{1}{\sigma \ell h_{n} \sqrt{-\log \left|h_{n}\right|}} O\left(h_{n}\right) \stackrel{n \rightarrow \infty}{\longrightarrow} 0
$$

we have

$$
\frac{\alpha\left(x+h_{n}\right)-\alpha(x)}{\sigma \ell h_{n} \sqrt{-\log \left|h_{n}\right|}}=\frac{1}{\sigma \ell \sqrt{-\log \left|h_{n}\right|}} \sum_{i=1}^{N\left(x, h_{n}\right)} \phi\left(f^{i-1}(x)\right)+r\left(x, h_{n}\right),
$$

where

$$
\lim _{n} \sup _{x \in[0,1]}\left|r\left(x, h_{n}\right)\right|=0 .
$$

From now on the proof is similar to an argument in Leplaideur and Saussol [26]. We will include it here for the sake of completeness. Let us define

$$
X_{N}(\theta, x)=\frac{1}{\sigma \sqrt{N}} \sum_{k=0}^{\lfloor N \theta\rfloor-1} \phi\left(f^{k}(x)\right)+\frac{(N \theta-\lfloor N \theta\rfloor)}{\sigma \sqrt{N}} \phi\left(f^{\lfloor N \theta\rfloor}(x)\right),
$$

and $Y_{n}$ by:

$$
Y_{n}(\theta, x)=\frac{1}{\sigma \sqrt{\nu_{n}(x)}} \sum_{k=0}^{\left\lfloor\nu_{n} \theta\right\rfloor-1} \phi\left(f^{k}(x)\right)+\frac{\left(\nu_{n}(x) \theta-\left\lfloor\nu_{n}(x) \theta\right\rfloor\right)}{\sigma \sqrt{\nu_{n}(x)}} \phi\left(f^{\left\lfloor\nu_{n}(x) \theta\right\rfloor}(x)\right)
$$

where $\nu_{n}(x)=N\left(x, h_{n}\right)$. By Lemma 3.1,

$$
\frac{N(x, h)}{-\log |h|} \stackrel{h \rightarrow 0}{\longrightarrow} \frac{1}{L}
$$

then

$$
\frac{N\left(x, h_{n}\right)}{-\log \left|h_{n}\right|} \stackrel{P}{\longrightarrow} \frac{1}{L} .
$$

By Lemma 2.7 and Keller [23] and Hofbauer and Keller [21] we have that $X_{N}(\theta, x)$ converges in distribution to the Wiener Process. We denote this convergence by

$$
X_{N}(\theta, x) \stackrel{D}{\longrightarrow}_{N} W .
$$

Then, [7, page 152] we conclude that

$$
Y_{n}(\theta, x) \stackrel{D}{\longrightarrow}_{n} W,
$$

where $W$ is the Wiener process. Hence, taking $\theta=1$ we conclude that

$$
Y_{n}(1, x) \stackrel{D}{\longrightarrow}_{n} \mathcal{N}(0,1),
$$

where $\mathcal{N}(0,1)$ denotes the Normal distribution with average zero and variance one. Observe that

$$
Y(1, x)=\frac{1}{\sigma \sqrt{\nu_{n}(x)}} \sum_{k=0}^{N(x, h)-1} \phi\left(f^{k}(x)\right) .
$$

Therefore, considering

$$
Z_{n}(x)=\frac{\sqrt{N\left(x, h_{n}\right)}}{\sqrt{-\log \left|h_{n}\right|}}
$$


by Slutsky's theorem (see [16]), since

$$
Z_{n} \stackrel{P}{\longrightarrow}_{n} \ell,
$$

we can conclude that

$$
\tilde{Y}_{n}(x)=Y_{n}(1, x) \cdot Z_{n}(x)=\frac{1}{\sigma \sqrt{-\log \left|h_{n}\right|}} \sum_{k=0}^{N(x, h)-1} \phi\left(f^{k}(x)\right) \stackrel{D}{\longrightarrow}_{n} \ell \mathcal{N}(0,1) .
$$

Hence, taking $R_{n}(x)=r\left(x, h_{n}\right)$ and using Slutsky's theorem one more time, we have

$$
\frac{1}{\ell} \tilde{Y}_{n}(x)+R_{n}(x)=\frac{\alpha\left(x+h_{n}\right)-\alpha(x)}{\sigma \ell h_{n} \sqrt{-\log \left|h_{n}\right|}} \stackrel{D}{\longrightarrow}{ }_{n} \mathcal{N}(0,1) .
$$

Proof of Corollary 1.7. The proof is identical to the proof of a similar statement in [29].

Proof of Theorem 1.8. By Keller [23] and Hofbauer and Keller [21] (see also Przytycki, Urbański and Zdunik [36] for the analytic setting ) we have that

$$
\limsup _{N \rightarrow \infty} \frac{\sum_{i=0}^{N-1} \phi\left(f^{i}(x)\right)}{\sqrt{2 N \log \log N}}=\sigma(\phi) .
$$

and

$$
\liminf _{N \rightarrow \infty} \frac{\sum_{i=0}^{N-1} \phi\left(f^{i}(x)\right)}{\sqrt{2 N \log \log N}}=-\sigma(\phi) .
$$

for $\mu$-almost every point $x$. By Proposition 2.3 and Lemma 3.1 the result easily follows.

\section{Dichotomy For the Regularity of $\alpha$}

We will prove Theorem 1.2 using methods similar to those in Heurteaux [20]. We need to introduce some notations and definitions. Given a function $w: \mathbb{R} \rightarrow \mathbb{R}$ we define the second-order difference of $w$ by

$$
\Delta_{h}^{2} w(x)=w(x+h)+w(x-h)-2 w(x) .
$$

Denote

$$
\omega(w, x, h)=\frac{\Delta_{h}^{2} w(x)}{|h|^{1+\epsilon}} .
$$

Lemma 4.1. We have

$$
\frac{\left|\Delta_{h}^{2} \alpha(x)\right|}{|h|^{1+\epsilon}}=|D f(x)|^{\epsilon} \frac{\left|\Delta_{D f(x) h}^{2} \alpha(f(x))\right|}{|D f(x) h|^{1+\epsilon}}+O(1) .
$$

Proof. In Corollary 1.1 we saw that $\alpha$ is in the Zygmund class $\Lambda_{1}$. So $\alpha$ is $\beta$-Hölder for every $0<\beta<1$ (see [13] and references therein). Consequently

$$
\begin{aligned}
\alpha(f(x+h)) & =\alpha\left(f(x)+D f(x) h+O\left(|h|^{2}\right)\right) \\
& =\alpha(f(x)+D f(x) h)+O\left(|h|^{1+\epsilon}\right) .
\end{aligned}
$$


on the other hand

$$
\begin{aligned}
\alpha(f(x+h)) & =v(x+h)+D f(x+h) \alpha(x+h) \\
& =v(x)+D v(x) h+D f(x) \alpha(x+h)+D^{2} f(x) \alpha(x+h) h+O\left(|h|^{1+\epsilon}\right) \\
& =v(x)+\left[D v(x)+D^{2} f(x) \alpha(x)\right] h+D f(x) \alpha(x+h)+O\left(|h|^{1+\epsilon}\right) .
\end{aligned}
$$

So

$$
\Delta_{D f(x) h}^{2} \alpha(f(x))=D f(x) \Delta_{h}^{2} \alpha(x)+O\left(|h|^{1+\epsilon}\right) .
$$

and (20) follows.

Therefore there is $K>0$ such that

$$
\omega(\alpha, x, h) \geq|D f(x)|^{\epsilon} \omega(\alpha, f(x), D f(x) h)-K,
$$

for every $x$ and $h \neq 0$. We will denote by $b=\inf \{|D f(x)|: x \in \mathbb{R}\}$ and $B=$ $\sup \{|D f(x)|: x \in \mathbb{R}\}$. It is easy to see that

Lemma 4.2. Let $K$ be as in (21). Let $x \in \mathbb{R}, h>0$ and $L>0$ satisfying

$$
\omega(\alpha, f(x), D f(x) h) \geq \frac{K+L}{b^{\epsilon}-1} .
$$

Then

$$
\omega(\alpha, x, h) \geq \frac{K+L|D f(x)|^{\epsilon}}{b^{\epsilon}-1},
$$

of Theorem 1.2. Note that

$$
\sup \{\omega(\alpha, x, h), x \in \mathbb{R}, h>0\}<\infty .
$$

if and only if $\alpha$ is of class $C^{1+\epsilon}$ (see [25, Lemma 5.4, page 207] for more details). Suppose that $\alpha$ is not $C^{1+\epsilon}$. Then there exists $x_{0} \in[0,1], h_{0} \in(0,1)$ and $L>0$ such that

$$
\omega\left(\alpha, x_{0}, h_{0}\right)>\frac{K+L}{b^{\epsilon}-1} .
$$

Given $n \in \mathbb{N}$, let $x_{i}$ be such that $f\left(x_{i+1}\right)=x_{i}$ for every $i<n$. Then by Lemma 4.2 we have

$$
\omega\left(\alpha, x_{n}, \frac{h_{0}}{D f^{n}\left(x_{n}\right)}\right) \geq \frac{L}{b^{\epsilon}-1}\left|D f^{n}\left(x_{n}\right)\right|^{\epsilon},
$$

that is

$$
\left|\Delta_{\frac{h_{0}}{D f^{n}\left(x_{n}\right)}}^{2} \alpha\left(x_{n}\right)\right| \geq \frac{L\left|h_{0}\right|^{\epsilon}}{b^{\epsilon}-1} \frac{h_{0}}{D f^{n}\left(x_{n}\right)} .
$$

Fix $y \in[0,1]$. For each $h>0$ let $n=n(h)$ be minimal such that

$$
f^{n}[y-h, y+h] \supset\left[x_{0}-h_{0}, x_{0}+h_{0}\right] .
$$

Then by Lemma 2.4 there is $C$ such that for every $h>0$ we have

$$
\frac{1}{C} \leq \frac{D f^{n}(x)}{D f^{n}(y)} \leq C
$$

and

$$
\frac{h}{C} \leq \frac{h_{0}}{D f^{n}(x)} \leq C h
$$


for every $x \in[y-h, y+h]$. Choose $x_{n} \in[y-h, y+h]$ such that $f^{n}\left(x_{n}\right)=x_{0}$. If $\alpha$ is differentiable at $y$ we have that

$$
\left|\Delta_{\frac{h_{0}}{D f^{n}\left(x_{n}\right)}}^{2} \alpha\left(x_{n}\right)\right|=o(h)=o\left(\frac{h_{0}}{D f^{n}\left(x_{n}\right)}\right),
$$

This contradicts $(22)$.

\section{REFERENCES}

[1] J. M. Anderson, J. Clunie, and C. Pommerenke. On Bloch functions and normal functions. J. Reine Angew. Math., 270:12-37, 1974.

[2] J. M. Anderson and L. D. Pitt. The boundary behavior of Bloch functions and univalent functions. Michigan Math. J., 35(2):313-320, 1988.

[3] J. M. Anderson and L. D. Pitt. Probabilistic behaviour of functions in the Zygmund spaces $\Lambda^{*}$ and $\lambda^{*}$. Proc. London Math. Soc. (3), 59(3):558-592, 1989.

[4] V. Baladi and D. Smania. Linear response formula for piecewise expanding unimodal maps. Nonlinearity, 21(4):677-711, (2008).

[5] V. Baladi and D. Smania. Smooth deformations of piecewise expanding unimodal maps. Discrete Contin. Dyn. Syst., 23(3):685-703, (2009).

[6] V. Baladi and D. Smania. Alternative proofs of linear response for piecewise expanding unimodal maps. Ergodic Theory Dynam. Systems, 30(1):1-20, (2010).

[7] P. Billingsley. Convergence of probability measures. Wiley Series in Probability and Statistics: Probability and Statistics. John Wiley \& Sons, Inc., New York, second edition, (1999). A Wiley-Interscience Publication.

[8] A. Broise. Transformations dilatantes de l'intervalle et théorèmes limites. Astérisque, (238):1109, (1996). Études spectrales d'opérateurs de transfert et applications.

[9] R. Burton and M. Denker. On the central limit theorem for dynamical systems. Trans. Amer. Math. Soc. vol. 302, 715-726 (1987).

[10] Z. Coelho and P. Collet. Asymptotic limit law for the close approach of two trajectories in expanding maps of the circle. Probab. Theory Related Fields 99 (1994), no. 2, 237-250.

[11] M. Denker, M. Gordin, and A. Sharova. A Poisson limit theorem for toral automorphisms. Illinois J. Math. 48 (2004), no. 1, 1-20.

[12] J. Donaire, J. Llorente, and A. Nicolau. Boundary values of harmonic gradients and differentiability of zygmund and weierstrass functions. Rev. Mat. Iberoam., 30(3):1037-1071, (2014).

[13] J. J. Donaire, J. G. Llorente, and A. Nicolau. Differentiability of functions in the Zygmund class. Proc. Lond. Math. Soc. (3), 108(1):133-158, (2014).

[14] N. G. Gamkrelidze. The modulus of continuity of the Weierstrass function. Mat. Zametki, 36(1):35-38, (1984).

[15] N. G. Gamkrelidze. On a probabilistic properties of Takagi's function. J. Math. Kyoto Univ., 30(2):227-229, (1990).

[16] A. Gut. Probability: a graduate course. Springer Texts in Statistics. Springer, New York, (2005).

[17] G. H. Hardy. Weierstrass's non-differentiable function. Trans. Amer. Math. Soc., 17(3):301325, (1916).

[18] M. Herman. Sur les courbes invariantes par les difféomorphismes de l'anneau. Vol. 2. Astérisque No. 144 (1986), 248 pp.

[19] M. Herman. Sur les courbes invariantes par les difféomorphismes de l'anneau. Vol. 1. With an appendix by Albert Fathi. With an English summary. Astérisque, 103-104. Société Mathématique de France, Paris, 1983. 221 pp.

[20] Y. Heurteaux. Weierstrass functions in Zygmund's class. Proc. Amer. Math. Soc., 133(9):2711-2720 (electronic), (2005).

[21] F. Hofbauer and G. Keller. Ergodic properties of invariant measures for piecewise monotonic transformations. Math. Z., 180(1):119-140, (1982).

[22] O. Ivrii. On Makarov's principle in conformal mapping. Preprint arXiv:1604.05619, 2016.

[23] G. Keller. Generalized bounded variation and applications to piecewise monotonic transformations. Z. Wahrsch. Verw. Gebiete, 69(3):461-478, 1985. 
[24] G. Keller and C. Liverani. Stability of the spectrum for transfer operators. Ann. Scuola Norm. Superiore Pisa 28: 141-52, 1999.

[25] S. G. Krantz. Lipschitz spaces, smoothness of functions, and approximation theory. Exposition. Math., 1(3):193-260, (1983).

[26] R. Leplaideur and B. Saussol. Central limit theorem for dimension of Gibbs measures in hyperbolic dynamics. Stoch. Dyn., 12(2):1150019, 24, (2012).

[27] P. Lévy. Théorie de l'addition des variables aléatoires. Gauthier-Villars, Paris, 1937

[28] P. Liardet and D. Volný. Sums of continuous and differentiable functions in dynamical systems. Israel J. Math. 98 (1997), 29-60.

[29] A. de Lima and D. Smania. Central limit theorem for the modulus of continuity of averages of observables on transversal families of piecewise expanding unimodal maps. Journal of the Institute of Mathematics of Jussieu, First View, Published online: 13 July 2016. https://doi.org/10.1017/S1474748016000177.

[30] A. N. Livšic. Cohomology of dynamical systems. Izv. Akad. Nauk SSSR Ser. Mat., 36:12961320, (1972).

[31] M. Lyubich. Feigenbaum-Coullet-Tresser universality and Milnor's hairiness conjecture. Ann. of Math. (2), 149(2):319-420, 1999.

[32] N. G. Makarov. On the distortion of boundary sets under conformal mappings. Proc. London Math. Soc. (3), 51(2):369-384, 1985.

[33] C. T. McMullen. Thermodynamics, dimension and the Weil-Petersson metric. Invent. Math., 173(2):365-425, 2008.

[34] W. Parry and M. Pollicott. Zeta functions and the periodic orbit structure of hyperbolic dynamics. Astérisque, (187-188):268, (1990).

[35] W. Philipp and W. Stout. Almost sure invariance principles for partial sums of weakly dependent random variables. Mem. Amer. Math. Soc. 2, (issue 2, 161):iv+140, (1975).

[36] F. Przytycki, M. Urbański, and A. Zdunik. Harmonic, Gibbs and Hausdorff measures on repellers for holomorphic maps. I. Ann. of Math. (2), 130(1):1-40, 1989.

[37] F. Przytycki, M. Urbański, and A. Zdunik. Harmonic, Gibbs and Hausdorff measures on repellers for holomorphic maps. II. Studia Math., 97(3):189-225, 1991.

[38] H. M. Reimann. Ordinary differential equations and quasiconformal mappings. Invent. Math., 33(3):247-270, (1976).

[39] T. Takagi. A simple example of the continuous function without derivative. Phys.-Math. Soc. Japan, 1:176-177, (1903).

[40] M. Viana. Stochastic dynamics of deterministic systems. Lecture notes XXI Colóquio Brasileiro de Matemática, IMPA, Rio de Janeiro, (1997).

Departamento de Matemática, iCMC-USP, Caixa Postal 668, CEP 13560-970, São Carlos-SP, Brazil.

E-mail address: smania@icmc.usp.br, amandal@icmc.usp.br

$U R L:$ http://conteudo.icmc.usp.br/pessoas/smania/ 